\title{
Effect of the quarternary ammonium compound trospium chloride on 24 hour jejunal motility in healthy subjects
}

T Schmidt, R Widmer, A Pfeiffer, H Kaess

\begin{abstract}
This study aimed to record 24 hour jejunal motility in healthy ambulant subjects and to analyse changes in motility caused by the oral administration of an anticholinergic agent, the quarternary ammonium compound, trospium chloride. In a placebo-controlled, double blind crossover trial, 24 hour jejunal motility was recorded in 12 healthy volunteers, aged 25 (2130) years, using a digital data logger connected to two strain-gauge transducers mounted 20 cm apart in a flexible nasojejunal catheter. A computer program was developed to determine contraction parameters. Trospium chloride (15 $\mathrm{mg}$ orally thrice daily) prolonged the duration of irregular contractile activity after meals $(p<0.02)$ and reduced its contraction frequency and amplitude $(p<0.001)$. In the fasting state, the cycle length of the migrating motor complex was prolonged $(p<0.01)$ by an extended phase I $(p<0.025)$. Phase III was shortened $(p<0.005)$ and showed a slower aboral migration velocity $(p<0 \cdot 005)$. Clustered contractions were less frequent during postprandial and fasting periods $(p<0 \cdot 01)$. Runs of clustered contractions were completely absent with trospium chloride. Digital manometry was useful for long term recordings of jejunal motility and enabled the motor effects of an anticholinergic agent to be characterised in ambulant subjects.
\end{abstract}

(Gut 1994; 35: 27-33)

Quarternary ammonium compounds are synthetic antimuscarinic agents widely taken orally as so-called antispasmodic drugs.' Their effect on human small bowel motility is largely unknown. It has even been doubted that these drugs have any effect when taken orally $^{2}$ because they are poorly absorbed from the intestine.'

It seems noteworthy that previous studies on this subject ${ }^{3-5}$ antedate the description of the migrating motor complex in humans. ${ }^{6}$ Moreover, these motility recordings were performed in fasting individuals and the observation periods were short. Finally, the methods used to study small bowel motility, balloon kymography and perfused tube manometry, can only be used in subjects who are immobilised in a laboratory where usual activities, normal food intake, or sleep are almost impossible.

Recent advances in the technology of small, catheter mounted pressure transducers ${ }^{7}$ and portable microcomputers have enabled ambulant recording systems for small bowel motility to be developed. ${ }^{8-11}$ These allow pro- longed studies, and subjects are largely able to maintain their usual life styles.

Trospium chloride (TC) is a quarternary ammonium compound (Fig 1) with a predominantly spasmolytic activity. ${ }^{1213}$ Other anticholinergic effects of the drug have been shown to be of little importance. ${ }^{12}{ }^{13}$ Its oral biovailability of about $3 \%{ }^{14}$ is in the same range as that described for other quarternary ammonium compounds ${ }^{1}$ but its spasmolytic activity in vitro, has been found to exceed that of hyoscine-N-butylbromide by a factor of $100 .^{12}$
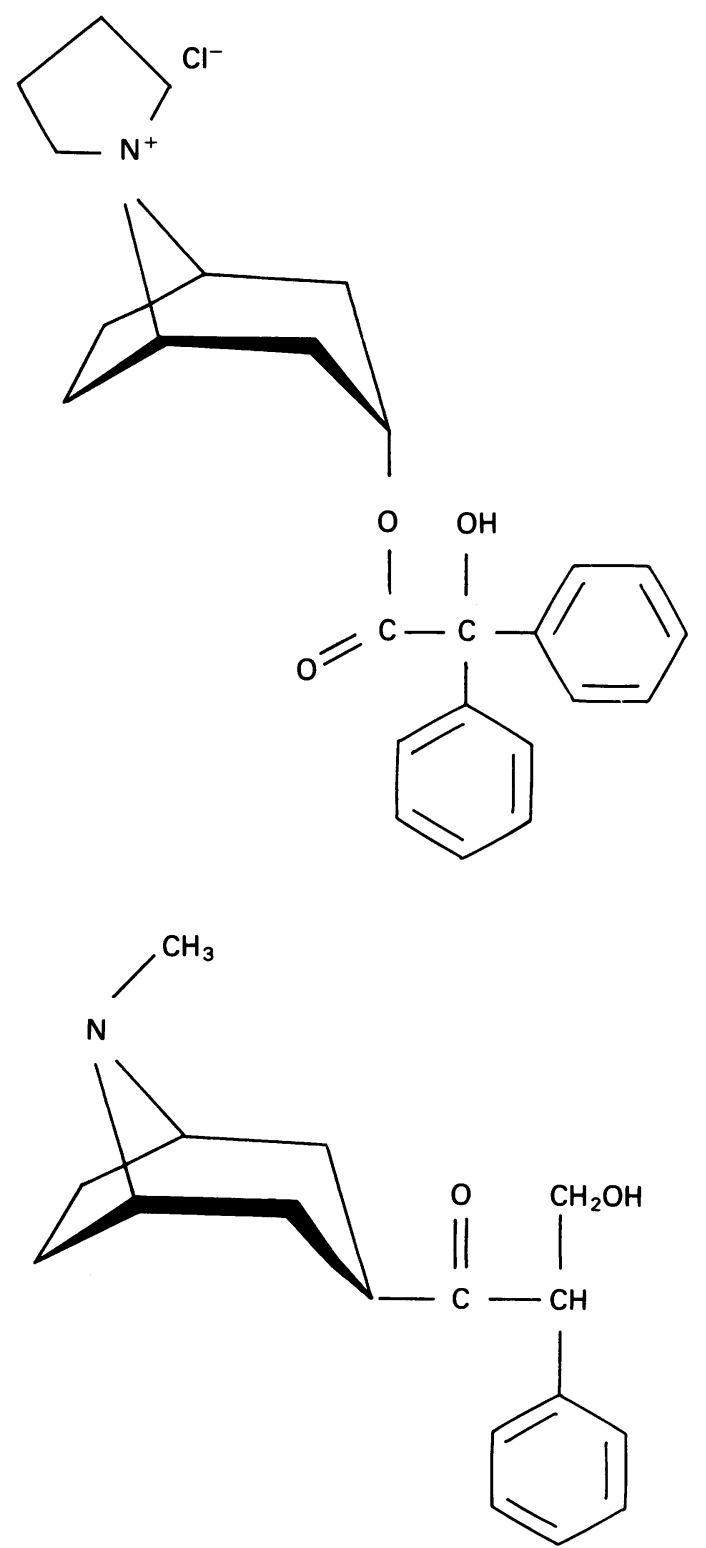

Figure 1: Structural formula of trospium chloride (top) in comparison with the classic antimuscarinic compound atropine (bottom). 
This study had the following aims: (a) to investigate 24 hour postprandial and fasting jejunal motility in healthy ambulant subjects and (b) to analyse the motor effects caused by oral administration of the quarternary ammonium compound TC.

\section{Subjects and methods}

\section{SUBJECTS}

Twelve healthy volunteers (six men, six women) aged $25(22-30)$ years participated in the study. All were non-smokers. None was taking any medication or had any history of gastrointestinal symptoms or surgery. The study was approved by the local ethical committee (Ethikkommission der Städtischen Krankenhäuser Münchens), and written informed consent was obtained from each subject.

\section{STUDY PROTOCOL}

The study was performed as a double-blind, placebo-controlled crossover trial. In a randomised order, each volunteer underwent two 24 hour recordings of jejunal motility, separated by a 'wash out' period of at least four weeks.

The subjects took $15 \mathrm{mg}$ placebo or TC (Spasmex, Dr Robert Pfleger Chemische Fabrik $\mathrm{GmbH}$, Bamberg, Germany) orally with tap water at $6 \mathrm{am}, 2 \mathrm{pm}$, and $10 \mathrm{pm}$ the day before and during the study period. This regimen was chosen because repeated oral doses of at least 10 mg TC are necessary to obtain effective plasma concentrations of about $1 \mathrm{ng} / \mathrm{ml} .{ }^{14} \mathrm{In}$ addition, we have shown previously ${ }^{15}$ that this oral dosage can influence gastrointestinal motility in humans. It significantly reduces gall bladder contraction in response to a meal, slows gastric emptying of a nutrient solution, and prolongs the orocoecal transit time.

On the examination day, the subjects fasted after a light breakfast taken before $8 \mathrm{am}$. Intubation of the small intestine began at $2 \mathrm{pm}$ (see 'recording system' for details) and was completed between 5 and $6 \mathrm{pm}$. At $6 \mathrm{pm}$ the motility recordings were started and the subjects, who were ambulant during the study, left the hospital to return at $6 \mathrm{pm}$ on the following day. The volunteers kept a diary in which their activities (which were not restricted during the recording period) were documented. The period of bed rest was scheduled from $10 \mathrm{pm}$ to $6 \mathrm{am}$ the next morning. Food intake was standardised according to current data on national diet habits ${ }^{16}$ and consisted of three mixed meals: dinner at $6.30 \mathrm{pm}$ (bread, cheese, sausages, fruit yoghurt; $679 \mathrm{kcal}$ ), breakfast at 8 am (bread, butter, marmalade, coffee; $478 \mathrm{kcal}$ ) and lunch at 12.30 pm (beef, mixed vegetables, potatoes, vanilla pudding; $699 \mathrm{kcal}$ ), providing a total of $1856 \mathrm{kcal}$ ( $18 \%$ proteins, $51 \%$ carbohydrates, $31 \%$ fat). Subjects were able to drink as much water as they liked.

To obtain data on the side effects of the placebo or TC, the subjects filled in a questionnaire after each recording. They had to indicate whether each of the following effects were present or not: dryness of mouth, absence of sweating, disturbances of accomodation and light sensitivity, increase in heart rate, disturbances of micturition. To judge side effects of the recording system, the subjects estimated their quality of sleep on a visual analogue scale from 0 to $100 \%{ }^{11} ; 100 \%$ corresponded to a 'normal' night for the individual.

DIGITAL RECORDING SYSTEM

Two piezoresistive intraluminal strain-gauge transducers (Keller AG für Druckmesstechnik, Winthertur, Switzerland) were used. The sensors had the following characteristics: linear range, $0-300 \mathrm{~mm} \mathrm{Hg}$; sensitivity, $5 \mu \mathrm{V} / \mathrm{V} /$ $\mathrm{mm} \mathrm{Hg}$; linearity and hysteresis error $\pm 0.4 \%$; nominal bridge resistance, $3.0 \mathrm{~K}$; temperature compensation, $20-39^{\circ} \mathrm{C}$. The sensors were incorporated at the distal end of a $210 \mathrm{~cm}$ flexible thermoplastic polyurethane catheter (OD 2.0 $\mathrm{mm}$ ), spaced $20 \mathrm{~cm}$ apart. The radio-opaque catheter was equipped with a free lumen communicating with a rubber balloon at its tip to facilitate intubation of the small intestine. The catheter was connected to a portable memory unit (PMT-Megalogger, Präzisionsmeßtechnik, Dr Ulrich Hoppe, Göttingen, Germany) that served as a power supply, preamplifier, and analogue to digital converter, providing a total storage capacity of 1.0 megabytes. Analogue to digital conversion was performed with a sampling rate of $3 \mathrm{~Hz}$ and a resolution of 8 bits for each sensor. The sampling rate was chosen in line with our own experiments (data not shown) and two recent systematic studies ${ }^{1011}$ that advocated sampling frequencies above $2 \mathrm{~Hz}$ in the small bowel to avoid missing contractions and falsely low values of contraction amplitudes. Before each measurement, the catheter was calibrated in a water bath at $37^{\circ} \mathrm{C}$ for 30 minutes so that a $34 \mathrm{~cm}$ column of water produced a $25 \mathrm{~mm} \mathrm{Hg}$ reading. No spontaneous baseline drifts or relevant noise phenomena were detectable during the calibration periods.

Intubation of the small intestine was performed transnasally after anaesthesia with $2 \%$ lidocaine aerosol spray (Xylocain Spray, Astra Chemicals, Wedel/Holstein, Germany) had been applied to nose and throat. Under fluoroscopic control, the tip of the catheter was advanced into the proximal duodenum. The rubber balloon was then filled with $4 \mathrm{ml}$ of a water soluble contrast medium (Ultravist R-300, Schering AG Pharma, Berlin, Germany) to enable the propulsive activity of the small intestine to pull the catheter aborally. The recording position was reached when the proximal sensor was located at the ligament of Treitz. The contrast medium was then re-aspirated, and the catheter was fixed by adhesive tape to the subject's nose. At $6 \mathrm{pm}$ the recording was started.

\section{DATA ANALYSES}

After each measurement the logger was unloaded to a personal computer (PCD-3Msx, Siemens AG, München, Germany) without data reduction. The pressure curves were analysed first by visual inspection: 
In each 24 hour recording, periods of fasting and fed state motility were determined. The fed state was defined as the period of irregular contractions from the beginning of each meal to the return of phase III activity, ${ }^{17}$ though other studies $^{818}$ have described a short 'phase I-like activity' indicating the return of fasting motility after meals, a finding that could not be identified in our recordings. As each meal interrupted the cyclic fasting activity for at least three hours in all experiments, this period was chosen to compare the effects of placebo or TC on postprandial contraction parameters. To obtain a global parameter for the digestive jejunal motor activity, a motility index defined as the total area under the contractions curve was calculated for the 30 minute period preceeding and for six 30 minute periods after each meal. The total duration of the fed state after a meal could only be compared after dinner in all individuals, since during the day, phase III activity did not return regularly before the beginning of the next meal.

Fasting motility was divided into three phases. ${ }^{6}$ Phase I was considered as the motor quiescence following a phase III. To avoid an arbitrary separation of phase I from phase II, phase I was defined as ending when the frequency of contractions exceeded for the first time, in either one or both channels, $10 \%$ of the maximum frequency during the migrating motor complex cycle at that recording point. ${ }^{19} \mathrm{Phase}$ III was defined as a series of uninterrupted contractions, at a rate of 10-12 per minute, and lasting at least two minutes showing either aboral progression or being present at the distal recording site only.$^{6}$ The migrating motor complex cycle length was taken as the time between the termination of two consecutive phase III activities at the distal sensor. ${ }^{20}$ The propagation velocity of phase III was calculated by dividing the distance between two sensors by the time interval needed for the onset of a phase III to traverse this distance. $^{6}$

It has become widely accepted that there are striking differences in the characteristics of diurnal and nocturnal migrating motor complex cycles $^{171821}$ necessitating a separate analysis. As only incomplete diurnal migrating motor complex cycles were observed in our experiments, the first phase III activity after dinner and all complete nocturnal cycles that occurred between dinner and breakfast on the following morning were used to compare the effects on fasting motility.

Discrete clustered contractions in the fasting and the fed states were identified by visual inspection of the tracings. Discrete clustered contractions were defined as groups of three to 10 regular contractions with amplitudes of $\geqslant 15 \mathrm{~mm}$ $\mathrm{Hg}$ occurring at a frequency of $1 / 5$ seconds, preceeded and followed by at least one minute of absent motor activity. ${ }^{22}$ Though discrete clustered contractions can move aborally, ${ }^{23}$ we did not try to analyse their migration as the distance between both sensors was too large to describe reliably the spatial and temporal relationships between the individual clusters in both channels.

To determine the contraction parameters during fasting and fed state motility (that is, frequency, amplitude, duration, and area under the curve of each contraction), and to calculate the motility index, a computer program had to be developed. ${ }^{24} 25$ Two representative 60 minute printouts of pressure curves (one with few artefacts, the other one with a great number of artefacts due to body movement and respiration) were analysed visually by four independent observers who were familiar with the recording technique. They were asked to classify each pressure event as either an artefact or as a contraction. In the case of a contraction, they had to determine its baseline level, its amplitude, and its duration. All pressure events that were regarded by three or more observers as a contraction (617 pressure events) were defined 'real contractions' and served as the standard for the development of a computer aided data evaluation method. Algorithms were then developed and gradually optimised in order to achieve as much agreement as possible with the results of visual analysis. Briefly, artefacts due to respiration and body movements were identified by their simultaneous occurrence and similar shape in both recording channels and were excluded from analysis. Only for peak recognition, digitally low pass filtered data were used $(0.26 \mathrm{~Hz}$ cut off frequency). Baseline level was defined as the pressure level at the beginning of each contraction and thresholds were set for amplitude $(\geqslant 9$ $\mathrm{mm} \mathrm{Hg}$ ), duration ( $\geqslant 2 \cdot 7$ seconds), and area under the curve $(\geqslant 20$ seconds $\times \mathrm{mm} \mathrm{Hg})$. Applying these algorithms, the computer was able to identify 'real contractions' in the representative tracings with a sensitivity of $91 \%$, a positive predictive value of $93 \%$, and a specificity of $99 \%$. When the mean values obtained by visual analysis were taken as $100 \%$, the mean contraction amplitude and duration determined by the computer program were $98.6 \%$, and $101.4 \%$, respectively.

All results are given as means (SEM). For statistical comparison a paired $t$ test was used. A $p$ value $<0.05$ was considered to be significant.

\section{Results}

The total duration of recording was $580 \cdot 7$ hours. Dryness of mouth the only side effect observed and was experienced by two subjects taking TC and two subjects taking placebo. The procedure was well tolerated, and apart from minor discomfort in the throat, no side effects due to the recording system were reported. Sleep quality was $89(4) \%$.

\section{EFFECTS ON FED STATE MOTILITY}

A schematic graph of all 24 hour recordings is given in Figure 2. The ingestion of each meal was immediately followed by a postprandial pattern of continuous random contractile activity that gradually decreased in frequency until the first postprandial phase III indicated the return of cyclical fasting activity. In the placebo studies the total duration of postprandial motility after dinner was 324 (29) minutes. With TC its duration was significantly $(p<0 \cdot 02)$ prolonged to 368 (27) minutes. A similar increase in the duration of fed state activity was found after 

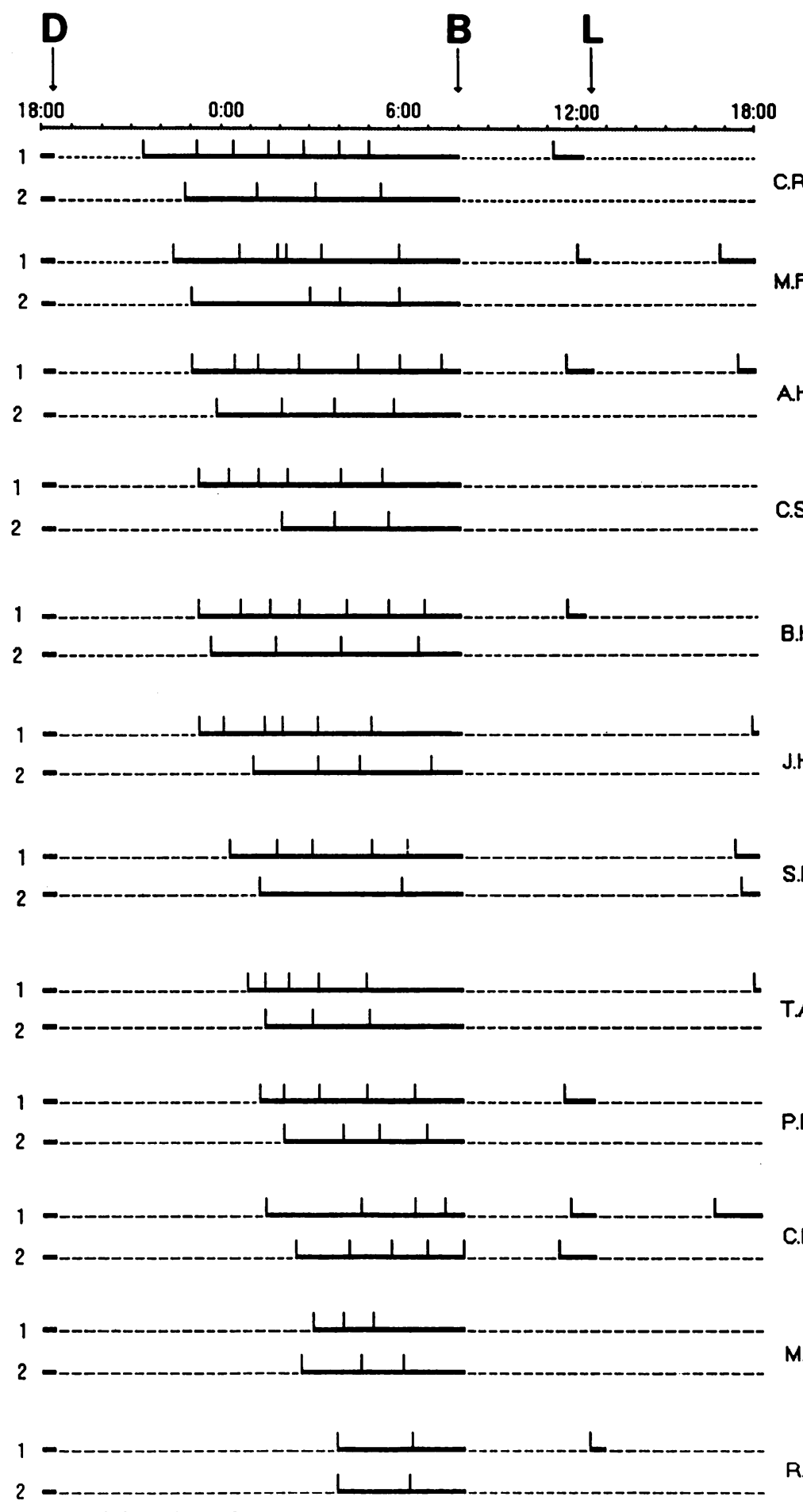

Figure 2: Schematic graph

of all 24 hour studies.
fejunal motility was

recorded from $6 \mathrm{pm}$ to $6 \mathrm{pm}$

on the following day in each

subject represented by initials on the right. The arrows indicate the beginning of meals; $D=$ dinner, $B=$ breakfast, $L=$ lunch; $1=$ recording with placebo; $2=$ recording with trospium chloride. The dashed line (-- ) signifies periods of postprandial motility, the solid line (-) periods of fasting motility. Phase III is represented by a vertical bar (卫).
TABLE I Parameters of individual jejunal contractions with placebo or trospium chloride in the fed state. One to three hours after meals (values, mean (SEM); $n=12$ )

\begin{tabular}{lccc}
\hline & Placebo & $\begin{array}{l}\text { Trospium } \\
\text { chloride }\end{array}$ & pValue \\
\hline Frequency $\left(\min ^{-1}\right)$ & $2 \cdot 24(0 \cdot 21)$ & $1 \cdot 08(0 \cdot 20)$ & $<0 \cdot 001$ \\
Amplitude $\left(\mathrm{mm} \mathrm{Hg}^{\star}\right)$ & $26 \cdot 5(1 \cdot 2)$ & $20 \cdot 3(0 \cdot 9)$ & $<0 \cdot 001$ \\
Duration $(\mathrm{s})$ & $3 \cdot 84(0.04)$ & $3 \cdot 79(0.06)$ & $\mathrm{NS}$ \\
Area $(\mathrm{mm} \mathrm{Hg} \times \mathrm{s})$ & $56 \cdot 4(2 \cdot 5)$ & $42 \cdot 4(2 \cdot 2)$ & $<0 \cdot 001$ \\
\hline
\end{tabular}

^Paired $t$ test.

NS= not significant.

breakfast and after lunch. After breakfast, there was a return of fasting activity before beginning lunch in seven subjects on placebo and in one subject on TC. Between lunch and the end of the recording, phase III activity returned in six placebo studies, whereas 11 individuals on TC remained in the fed state (Fig 2). The time course of the motility index over the 30 minute period before and the 180 minute period after dinner, breakfast, and lunch is shown in Figure 3. In the placebo studies, the ingestion of each meal was followed by a steep increase in the motility index within 30 minutes, and the index remained significantly high for 150 minutes ( $p$ values between 0.05 and 0.005 ) when compared with the 30 minute preprandial period. After 180 minutes, the preprandial values were reached. In the TC studies, there was a significantly $(p<0.001)$ lower motility index both before and after eating, and no changes with time were apparent over the three postprandial hours.

The influences of TC on the parameters of TA individual postprandial contractions responsible for the differences in the motility index are summarised for all three meals in Table I. TC induced a significant $(\mathrm{p}<0.001)$ reduction in the frequency, amplitude, and area of the individual postprandial contractions. The duration of contractions remained unchanged compared with placebo. In accordance with the results of the motility index, there were no significant differences in the contraction parameters obtained after dinner, breakfast, and lunch.

Discrete clustered contractions could be identified postprandially in each placebo study (Fig 4 (A)). The total number of clusters was 42 (12) in 24 hours, and they were randomly distributed throughout the postprandial periods. Altogether $69 \cdot 7 \%$ of discrete clustered contractions were isolated clusters; $30.3 \%$ occurred in runs of two to eight consecutive clusters at a mean frequency of $1 \cdot 2(0 \cdot 1)$ per minute. With
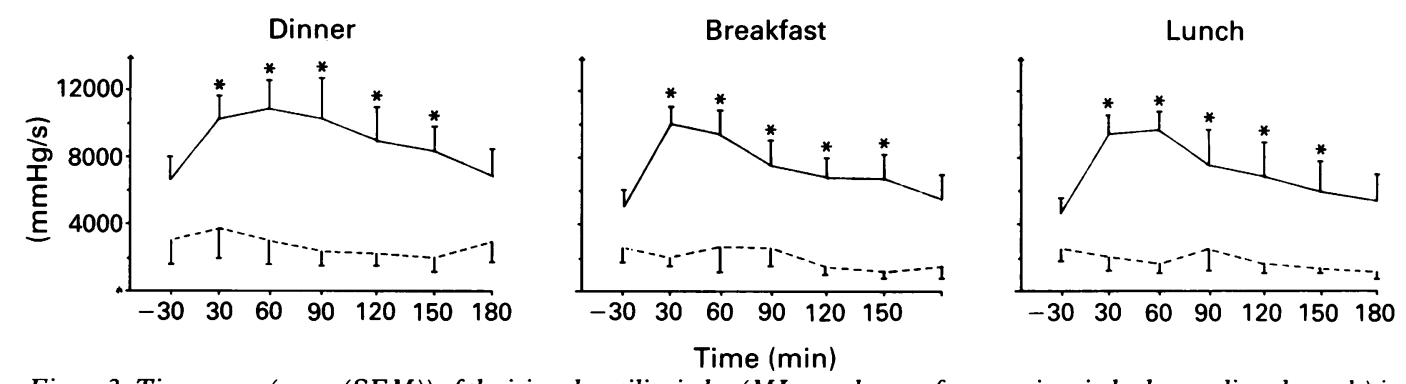

Figure 3: Time course (mean (SEM)) of the jejunal motility index (MI=total area of contractions in both recording channels) in the 30 minute period before, and in six 30 minute periods after dinner, breakfast, and lunch. With placebo (solid lines), each meal was followed by a significant increase $\left({ }^{\star} p\right.$ values between $<0.05$ and 0.005$)$ of $M I$ for 150 minutes when compared with the preprandial level. With trospium chloride preprandial and postprandial MIs were significantly $(p<0 \cdot 001)$ lower than with placebo, and no significant changes were seen over the three postprandial hours. 

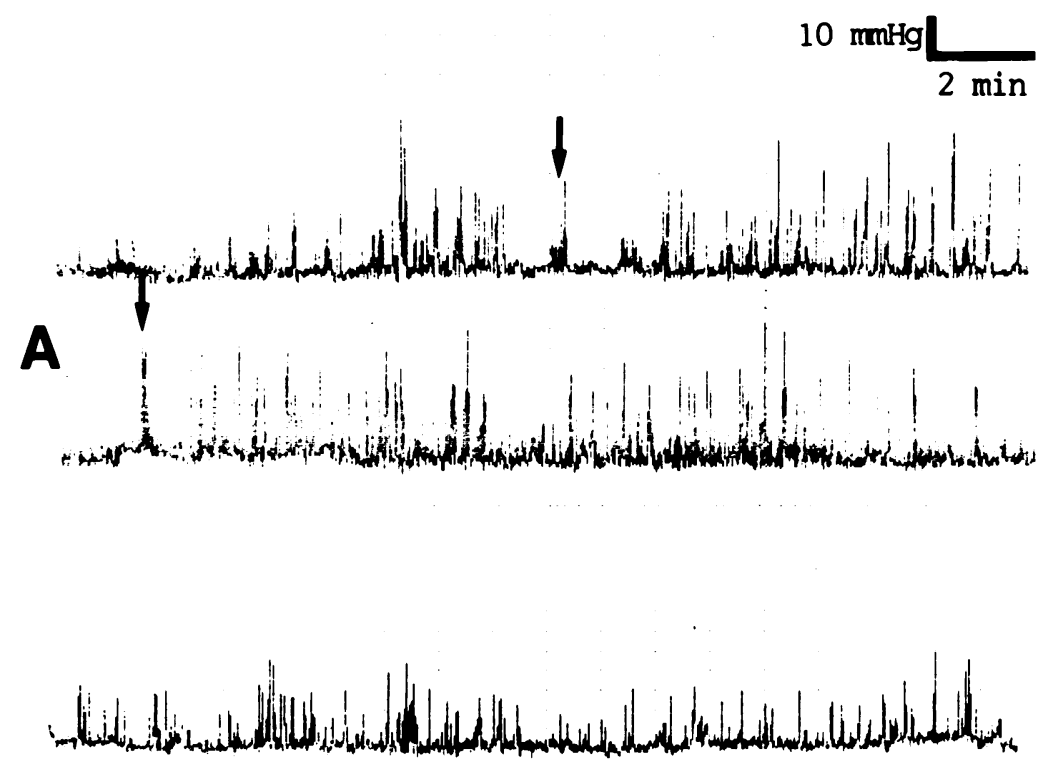

B

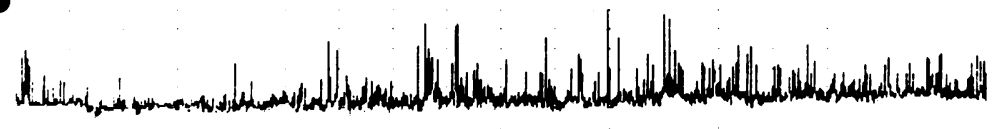

Figure 4: An example of postprandial jejunal motility with placebo $(A)$ and trospium chloride $(B)$ in the same subject $(I H)$ one hour after breakfast. With trospium chloride, a reduction in the frequency and amplitude of postprandial contractions was observed. Two isolated discrete clustered contractions in the placebo tracing are marked by arrows. These are absent in the trospium chloride tracing.
TABLE III Parameters of individual jejunal contractions in the fasting state (values, mean $(S E M) ; n=12$ )

\begin{tabular}{|c|c|c|c|}
\hline & Placebo & $\begin{array}{l}\text { Trospium } \\
\text { chloride }\end{array}$ & $p$ Value ${ }^{\star}$ \\
\hline $\begin{array}{l}\text { Phase II: } \\
\text { Frequency }\left(\mathrm{min}^{-1}\right) \\
\text { Amplitude }(\mathrm{mm} \mathrm{Hg} \mathrm{Hg}) \\
\text { Duration }(\mathrm{s}) \\
\text { Area }(\mathrm{mm} \mathrm{Hg} \times \mathrm{s})\end{array}$ & $\begin{array}{c}1 \cdot 37(0.09) \\
22 \cdot 6(0 \cdot 9) \\
3 \cdot 64(0 \cdot 12) \\
46 \cdot 7(1 \cdot 6)\end{array}$ & $\begin{array}{c}1 \cdot 32(0 \cdot 10) \\
21 \cdot 7(0 \cdot 6) \\
3 \cdot 49(0 \cdot 10) \\
44 \cdot 3(2 \cdot 8)\end{array}$ & $\begin{array}{l}\text { NS } \\
\text { NS } \\
\text { NS } \\
\text { NS }\end{array}$ \\
\hline $\begin{array}{l}\text { Phase III: } \\
\text { Frequency }\left(\mathrm{min}^{-1}\right) \\
\text { Amplitude }\left(\mathrm{mm} \mathrm{Hg}^{\mathrm{H}}\right) \\
\text { Duration }(\mathrm{s}) \\
\text { Area }(\mathrm{mm} \mathrm{Hg} \times \mathrm{s})\end{array}$ & $\begin{array}{c}10 \cdot 6(0 \cdot 3) \\
27 \cdot 6(1 \cdot 9) \\
3.72(0 \cdot 05) \\
57 \cdot 3(4 \cdot 1)\end{array}$ & $\begin{array}{c}10 \cdot 6(0 \cdot 3) \\
25 \cdot 3(1 \cdot 7) \\
3 \cdot 72(0 \cdot 08) \\
52 \cdot 8(3 \cdot 5)\end{array}$ & $\begin{array}{l}\text { NS } \\
\text { NS } \\
\text { NS } \\
\text { NS }\end{array}$ \\
\hline
\end{tabular}

$\star$ Paired $t$ test.

NS $=$ not significant

during phase II and III activity are shown in Table III. In contrast to the fed state, the frequency, amplitude, duration, and area of individual contractions during phase II and phase III did not show any differences between placebo and TC.

In the fasting state, discrete clustered contractions were observed only during late phase II activity. With placebo and TC, discrete clustered contractions were less frequent during fasting motility compared with the fed state $(\mathrm{p}<0.025)$. They were present in all individuals on placebo and showed a mean number of 11 (1). Altogether $79 \cdot 1 \%$ of clusters with placebo were isolated clusters, $20.9 \%$ occurred in runs of two to eight clusters with the same frequency, as seen postprandially (Fig $5(\mathrm{~A})$ ). With TC, clustered contractions in the fasting state were completely absent in five individuals. The mean number of clusters decreased to 4 ( 1 ) in 24 hours $(p<0 \cdot 01)$. Again, only isolated discrete clustered contractions and no runs were seen with $\mathrm{TC}$ in the fasting state (Fig 5 (B)).

\section{Discussion}

As shown in Figure 2, a significant $(p<0.005)$ reduction in the mean number of nocturnal phase III activities from $5 \cdot 3(0.5)$ with placebo to $3.5(0.3)$ with TC occurred. This effect caused a significant $(p<0.01)$ increase in the migrating motor complex cycle length. In addition, differences in the duration of the migrating motor complex phases were observed and these are summarised in Table II. Compared with placebo, TC prolonged the migrating motor complex cycle length due to a longer duration of phase I $(p<0.025)$. Phase III was shortened after TC $(p<0.005)$ and showed a slower $(p<0.005)$ aboral migration velocity (Fig 5 ). No effect was observed on the duration of phase II.

The parameters of individual contractions

TABLE II Effects of placebo or trospium chloride on the migrating motor complex cycle (values, mean (SEM); $n=12$ )

\begin{tabular}{lccc}
\hline & Placebo & $\begin{array}{l}\text { Trospium } \\
\text { chloride }\end{array}$ & $p$ Value $^{\star}$ \\
\hline Cycle length (min) & $77(6)$ & $116(11)$ & $<0.01$ \\
Phase I duration (min) & $42(4)$ & $78(12)$ & $<0.025$ \\
Phase II duration (min) & $38(6)$ & $34(6)$ & NS \\
Phase III duration(min) & $7.3(0.8)$ & $3.8(0.3)$ & $<0.005$ \\
Velocity (cm/min) $\dagger$ & $8 \cdot 4(0.6)$ & $4.7(0.4)$ & $<0.005$ \\
\hline
\end{tabular}

${ }^{\star}$ Paired $t$ test.

†Aboral migration velocity of phase III

NS=not significant.
In this study, the effects of an orally administered antimuscarinic agent on 24 hour jejunal motility in healthy humans were characterised. Anticholinergic agents with a quarternary ammonium structure are unselective competitive antagonists of acetylcholine at muscarinic receptors, ' of which the $M_{1}$ subtype is present on myenteric neurons and smooth muscle cells and the $M_{3}$ subtype is present on smooth muscle cells only. ${ }^{26}$

With regard to the effects on postprandial motility, our finding that TC reduced the frequency and amplitude of postprandial jejunal contractions is in line with current views of peripheral cholinergic transmission in the small bowel. ${ }^{23}$ To our knowledge, however, there are no published data on the effects of an orally administered quarternary ammonium compound on postprandial small bowel motility, ${ }^{2}$ despite their widespread oral use as so called antispasmodics.

The mean duration of postprandial motility in our placebo studies agrees with the results obtained by others with ambulant manometry. ${ }^{111}$ The prolongation of the fed state period with TC may be explained by two mechanisms: an effect on migrating motor complex cycling that will be discussed below, and an effect of TC on gastric emptying. The duration 


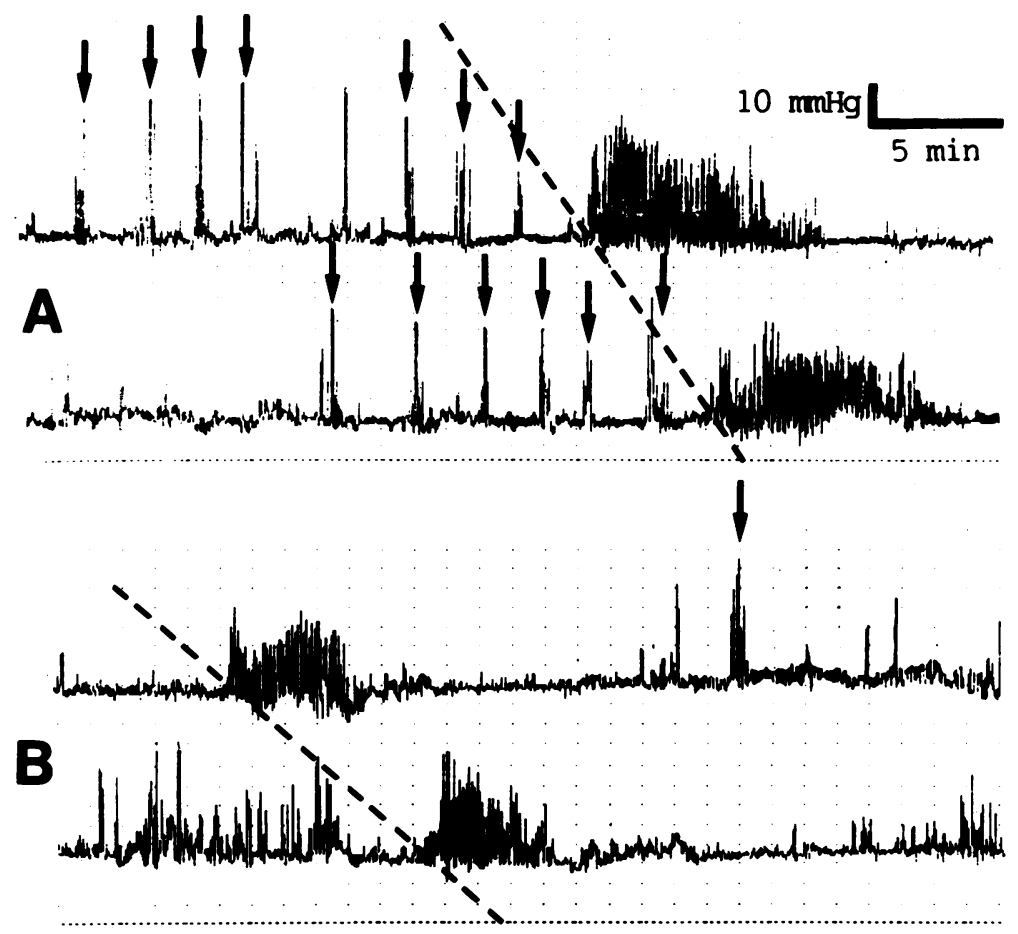

Figure 5: An example of fasting jejunal motility with placebo $(A)$ and trospium chloride $(B)$ in the same subject $(M F)$. This subject had a phase III at 6 am in both recordings (see Fig 2). With trospium chloride,

phase III was shortened and had a slower aboral migration velocity. Series of discrete clustered contractions (arrows) were preceeding phase III with placebo. With trospium chloride, runs of discrete clustered contractions were absent. An isolated discrete clustered contraction (arrow) was observed in the ongoing phase II of the next migrating motor complex cycle (arrow). of the fed state has been shown to be closely correlated with the entire gastric emptying time. ${ }^{27}$ We observed that TC slows gastric emptying of a nutrient solution in healthy subjects. ${ }^{15}$ Oral cyclotropium bromide and hyosine N-butyl-bromide, two other quarternary ammonium compounds, have been shown to delay gastric emptying of a semisolid meal. ${ }^{28}$ In addition, a correlation between the half time for gastric emptying and the motility index in the small bowel after a solid meal has been shown. ${ }^{29}$ Therefore, it cannot be excluded that different rates of gastric emptying have contributed to the different time courses of the postprandial motility index in our study.

Postprandial discrete clustered contractions were initially described in partial mechanical small bowel obstruction, ${ }^{22}$ but were absent in healthy controls during a perfused tube recording for one to two hours after a solid meal. In our 24 hour studies with placebo, discrete clustered contractions could be identified as part of the digestive jejunal motility in each healthy subject. Most, however, were isolated, and they were not the predominant feature of postprandial motility. It was shown that the occurrence of this distinct postprandial motor pattern can be reduced by the administration of an antimuscarinic compound.

With regard to the effects on fasting motility, it has already been shown that antimuscarinic drugs are able to modulate the neural circuitries governing the migrating motor complex cycle, ${ }^{18}{ }^{30} 31$ but the data are inconsistent. In dogs, unselective cholinergic blockade with atropine, in high doses of $30 \mu \mathrm{g} / \mathrm{kg}$ body weight intravenously, significantly prolonged the jejunal migrating motor complex cycle length and phase I duration, and reduced the migration velocity of phase III. ${ }^{30}$ In the human ileum, atropine given as a bolus of $7 \mu \mathrm{g} / \mathrm{kg}$ body weight intravenously has been shown to induce phase III activity in the fasting and in the fed states, ${ }^{31}$ an effect that was not observed in our jejunal recordings. These examples illustrate that the effects of antimuscarinic drugs on small bowel motility may depend on the dose, how the drug is given, and the region of the small intestine. Furthermore, antimuscarinic effects on contraction parameters may not be identical in the fed and the fasting states: postprandially, TC reduced the frequency and amplitude of contractions, whereas no effect on these parameters was observed in phases II and III motor activity.

Recently, repetitive clustered contractions, ${ }^{182-34}$ especially runs of discrete clustered contractions ranging from seven to 190 minutes ${ }^{18}$ and occurring in a 'minute rhythm', have been reported as a characteristic feature of diurnal phase II activity in the irritable bowel syndrome, and these runs showed some association with typical episodes of pain. ${ }^{18}{ }^{32}$ Our placebo experiments confirm previous work ${ }^{18}$ in that series of more than eight discrete clustered contractions are absent in healthy subjects. Though our observation that TC reduced the occurrence of discrete clustered contractions during phase II is largely based on nocturnal recordings, further studies are necessary to clarify whether the same effects can be obtained in patients with the irritable bowel syndrome during diurnal phase II, and whether this is associated with relief of their symptoms. Recently, a three month, double-blind, placebo-controlled study of the quarternary ammonium compound cimetropium bromide showed sustained alleviation of abdominal pain in irritable bowel syndrome patients. ${ }^{35}$

Finally, in this study digital manometry was used, a method that has been shown to produce data that are closely comparable with those acquired from perfused tube manometry under laboratory conditions. ${ }^{9}$ Together with a computer aided data evaluation that was carefully compared with visual analysis, it provided detailed quantitative data on small bowel motility in subjects following their daily activities in their usual environment.

In conclusion, the antimuscarinic compound TC (15 mg orally thrice daily) can reduce jejunal motor activity in healthy subjects with no relevant side effects. After meals, the duration of the fed pattern is prolonged and the frequency and amplitude of its contractions are reduced. In the fasting state, migrating motor complex cycle composition is mainly modified by a longer duration of motor quiescence. The reduction of clustered activity may be of interest in the treatment of patients with the irritable bowel syndrome. Digital manometry proved to be a suitable method for prolonged pharmacological studies of small bowel motility in ambulant subjects.

This work was supported by Dr Robert Pfleger Foundation, Bamberg, Germany.

The authors are indebted to Professor David L Wingate, London, for his fruitful comments and criticism of this paper.

Part of this work was presented as a poster at the 13th International Symposium on Gastrointestinal Motility, Kobe, Japan, 1991 and was published in abstract form: $\mathcal{F}$ Gastroint Motility 1991; 3: 199.

1 Brown JH. Atropine, scopolamine, and related antimuscarinic drugs. In: Gilman AG, Godman LS, eds. The pharmacological basis of therapeutics. 8th Ed. New York: MacMillan Publishing, 1990: 150-65. 
2 Ivy KJ. Are anticholinergics of use in the irritable colon syndrome? Gastroenterology 1975; 68: 130-7.

3 Chapman WP, Rowlands EN, Jones CM. Multiple-baloon kymographic recording of the comparative action of atropine, tincture of belladonna and placebos on the motility
of the upper small intestine in man. $N E$ El $F$ Med 1950; 233: 243-8.

4 Schmid E, Ritter U. The effect of rectally administered hyoscine-N-butylbromide on the motility of the small intestine. Drugs made in Germany 1972;15: 7-10.

5 Miyoshi A, Suyama T, Karamura I. A double-blind comparative study of the inhibitory effect of intraduodenally tive study of the inhibitory effect of intraduodenally motility. F Int Med Res 1977; 5: 223-32.

6 Vantrappen G, Janssens J, Hellemans J, Ghoos Y. The interdigestive motor complex of normal subjects and patients with bacterial overgrowth of the small intestine. $\mathcal{f}$ Clin Invest 1977; 59: 1158-66.

7 Mathias JR, Sninsky CA, Millar HD, Clench MH, Davis RH. Development of an improved multi-pressure-sensor probe for recording muscle contraction in human intestine. Dig Dis Sci 1985; 30: 119-23.

8 Gill RC, Kellow JE, Wingate DL. The migrating motor complex at home. Gastroenterology 1987; 92: 1405

9 Gill RC, Kellow JE, Browning C, Wingate DL. The use of intraluminal strain gauges for recording ambulant small bowel motility. Am $\mathscr{f}$ Physiol 1990; 258 (Gastrointest Liver Physiol 21): G610-5.

10 Lindberg G, Iwarzon M, Stal P, Seensalu R. Digital ambulatory monitoring of small-bowel motility. Scand $\mathcal{f}$ Gastroenterol 1990; 25: 216-24.

1 Husebye E, Skar V, Aalen O, Osnes M. Digital ambulatory manometry of the small intestine in healthy adults. Dig Dis Sci 1990; 35: 1057-67.

12 Antweiler H, Lauterbach F, Lehmann HD, Vebel H, Vogel G. Zur Pharmakologie und Toxikologie von AzoniaspiroVerbindungen in der Nortropin- bzw. PseudonortropinReihe. Arzneim Forsch/Drug Res 1966; 16: 1581-91.

13 Bertold H, Pfleger R, Schulz W. Über Azonia-spiroVerbindungen. Arzneim Forsch/Drug Res 1967; 17: 714-9.

14 Schladitz-Keil G. Spahn H, Mutschler E. Determination of the bioavailability of the quarternary ammonium compound trospium chloride in man from urinary excretion data. Arzneimittel-forschung (Aulendorf) 1986; 36: 984-7.

15 Pfeiffer A, Schmidt T, Höller T, Hermann H, Wendl B, Pehl $\mathrm{C}$, Kaess $\mathrm{H}$. The effect of trospium chloride on gastrointestinal motility in man. Eur $\mathcal{f}$ Clin Pharmacol 1993; 47: 219-23.

16 Ermährungsbericht 1988. Frankfurt: Deutsche Gesellschaft für Ernährung, 1988: 259-308.

17 Kellow JE, Borody TE, Phillips SF, Tucker RL, Haddad AC. Human interdigestive motility: variations in patterns from Human interdigestive motility: variations in patterns

18 Kellow JE, Gill RC, Wingate DL. Prolonged ambulan recordings of small bowel motility demonstrate abnormalities in the irritable bowel syndrome. Gastroenterology 1990; 98: 1208-18.
19 Sarna SK, Soergel KH, Harig JM, Loo FD, Loo CM, Donahue KM, et al. Spatial and temporal patterns of human jejunal contractions. Am f P hysiol 1989; 257: G423-32.

20 Rees WDW, Maladelada JR, Miller LJ, Go VLW. Human interdigestive and postprandial gastrointestinal motor and gastrointestinal hormone patterns. Dig Dis Sci 1982; 27: 321-9.

21 Kumar D, Wingate DL, Ruckebusch Y. Circadian variation in the migration velocity of the migrating motor complex. Gastroenterology 1986; 91: 926-30.

22 Summers RW, Anuras, Green J. Jejunal manometry patterns in health, partial intestinal obstruction and pseudoobstrucin health, partial intestinal obstruction and

23 Sarna SK, Otterson MF. Small intestinal physiology and pathophysiology. Gastroent Clin North Am 1989; 18: 375404

24 Benson MJ, Castillo FD, Demitrakopoulos J, Spyron NM, Wingate DL. Automated analysis of prolonged ambulatory small intestinal data: breaking the 'code'? Gastroenterology 1991; 100: A420.

25 Benson MJ, Castillo FD, Deeks JJ, Wingate DL. Assessment by prolonged ambulatory manometry of the effect of oral cisapride on proximal small bowel inter-digestive motility. Dig Dis Sci 1992; 37: 1569-75.

26 Goyal RK. Muscarinic receptor sub types. Physiology and clinical implications. N Engl f Med 1989; 321: 1022-9.

27 Madsen JL, Dahl K. Human migrating myoelectric complex in the relation to gastrointestinal transit of a meal. Gut 1990 ; 31: $1003-6$.

28 Stacher G, Bergmann H, Havlik E, Schmierer G, Schneider C. Effects of oral cyclotropium bromide, hyoscine $\mathrm{N}$-butylbromide and placebo on gastric emptying and antral motor activity in healthy man. Gut 1984; 25: 485-90.

29 Read NW, Al-Janabi MN, Edwards CA, Barber DC. Relationship between postprandial motor activity in the human small intestine and the gastro-intestinal transit of food. Gastroenterology 1984; 86: 721-7.

30 Schiavone A, Sagrada A, Pagani F, Giachetti A. Role of muscarinic receptor subtypes in the regulation of migrating myoelectric complex in the dog. Gastroenterology 1989; 96: 116-20.

31 Borody TJ, Quigley EMM, Phillips SF, Wienbeck M, Tucker RL. Effects of morphine and atropine on motility and transit in the human ileum. Gastroenterology 1985; 89: 562-70.

32 Kellow J. Phillips SF. Altered small bowel motility in irritable bowel syndrome is correlated with symptoms. Gastroenterology 1987; 92: 1885-92.

33 Kellow JE, Eckersley G, Jones MP. Enhanced perception of physiological intestinal motility in the irritable bowel syndrome. Gastroenterology 1991; 101: 1621-7.

34 Kellow JE, Eckersley GM, Jones M. Enteric and central contribution to intestinal dysmotility in irritable bowel contribution to intestinal dysmotility
syndrome. Dig Dis Sci 1992; 37: 168-74.

35 Dobrilla G, Imbimbo BP, Piazzi L, Bensi G. Longterm treatment of irritable bowel syndrome with cimetropium bromide: a double-blind placebo-controlled trial. Gut 1990 ; 31: $335-58$. 